\title{
Analytic validity of DecisionDx-Melanoma, a gene expression profile test for determining metastatic risk in melanoma patients
}

Robert W. Cook ${ }^{1 *}$, Brooke Middlebrook ${ }^{1}$, Jeff Wilkinson², Kyle R. Covington ${ }^{1}$, Kristen Oelschlager², Federico A. Monzon ${ }^{1}$ and John F. Stone ${ }^{2}$

\begin{abstract}
Background: The DecisionDx-Melanoma test provides prognostic information for patients with cutaneous melanoma (CM). Using formalin-fixed paraffin-embedded primary tumor tissue, the RT-PCR-based test classifies patients into a low- (Class 1) or high-risk (Class 2) category for recurrence based on expression of 31 genes. The current study was designed to assess the analytical validity of this test.

Methods: Inter-assay, inter-instrument, and inter-operator studies were performed to evaluate reliability of the 31gene expression test results, sample stability and reagent stability. From March 2013 through June 2016, the gene expression test was performed on 8244 CM tumors. De-identified data from Pathology Reports were used to assess technical success.

Results: Robust sample and reagent stability was observed. Inter-assay concordance on 168 specimens run on 2 consecutive days was $99 \%$ and matched probability scores were significantly correlated $\left(R^{2}=0.96\right)$. Inter-instrument concordance was $95 \%$, and probability scores had a correlation $R^{2}$ of $0.99(p<0.001)$. From 8244 CM specimens submitted since 2013, 85\% (7023) fulfilled pre-specified tumor content parameters. In these samples with sufficient tumor requirements, the technical success of the test was $98 \%$.
\end{abstract}

Conclusion: DecisionDx-Melanoma is a robust gene expression profile test that demonstrates strong reproducibility between experiments and has high technical reliability on clinical samples.

Keywords: Gene expression profiling, DecisionDx-Melanoma, Cutaneous melanoma, Metastasis, Analytic validity, Technical success

\section{Background}

Clinical staging of cutaneous melanoma $(\mathrm{CM})$ is based upon clinicopathologic parameters such as tumor thickness, ulceration, mitotic rate, and sentinel lymph node (SLN) status [1]. SLN status, as determined by SLN biopsy (SLNB), has the greatest prognostic significance of established factors [2-4]. The majority of CM patients are initially diagnosed with early stage (I or II) disease and have favorable prognosis $[1,5]$. However, a

\footnotetext{
*Correspondence: rcook@castlebiosciences.com

${ }^{1}$ Castle Biosciences, Inc., 820 S. Friendswood Dr., Suite 201, Friendswood, TX 77546, USA

Full list of author information is available at the end of the article
}

substantial percentage of early stage patients develop metastases and two-thirds of all melanoma-related deaths occur in patients initially diagnosed with Stage I or II disease [1, 5-7]. Accurate methods for predicting metastatic risk are, therefore, of paramount importance for implementing risk appropriate management plans to enable early identification of disease progression and timely intervention with current treatment options.

We have developed and validated a gene expression profile (GEP) test that assesses melanoma tumor biology to improve the prediction of metastasis risk beyond traditional clinicopathologic factors $[8,9]$. The GEP test employs RT-PCR gene expression analysis to evaluate the 
expression of 31 gene targets using primary tumor biopsy tissue and provides a binary classification of low risk (Class 1) or high risk (Class 2) of metastasis within 5 years. Clinical validation studies have shown the test to be an accurate prognosticator that is independent of AJCC staging criteria $[8,9]$. Molecular classification was shown to improve risk prediction when used in combination with SLNB, identifying as Class 2 more than $80 \%$ of SLN-negative patients who developed metastatic disease and died from melanoma [8].

In this study, we report the analytic validity of the GEP test, including reproducibility (inter-assay, interinstrument, and inter-operator concordance) of molecular classification and technical reliability of clinical testing in accordance with published guidelines [10, 11], when performed in a College of American Pathologists (CAP)-accredited, Clinical Laboratory Improvement Act (CLIA)-certified laboratory setting.

\section{Methods}

\section{Sample and clinical data collection}

All samples were acquired through routine clinical testing of primary CM tumors with the 31-GEP test. Ten 5$\mu \mathrm{m}$ tissue sections were cut from the formalin-fixed, paraffin-embedded (FFPE) block containing the primary melanoma tissue (biopsy or wide local excision). The first recut slide was stained with hematoxylin and eosin (H\&E) and the 9 subsequent slides remained unstained. The slides were sent to Castle Biosciences' centralized CAP-accredited, CLIA-certified laboratory. All analyses were performed using de-identified technical and pathology report data. Therefore, Institutional Review Board approval was not required because this analysis is exempt from the regulatory review requirements as set forth in section 46.101 (b) of 45 CFR 46.

\section{RT-PCR analysis and risk assignment}

The H\&E-stained slides for all research and clinical samples are reviewed by a licensed pathologist for (a) confirmation of the presence of primary melanoma tumor and (b) marking an area containing sufficient tumor density (initially $\geq 60 \%$ and subsequently lowered to $\geq 40 \%$ ). On the unstained slides, this tumor tissue was macrodissected and cDNA was converted from total RNA as previously described [9]. Quantitative real-time PCR was performed on the 7900HT Fast Real Time PCR System (Life Technologies) or the QuantStudio OpenArray system (Life Technologies). Cards or OpenArrays contained primers specific for 28 class-discriminating gene targets and three endogenous control genes [9].

Research cases used for stability studies had RNA extracted only once per sample (Fig. 1). RNA stability studies, therefore, compared assays run from the same isolated RNA sample that were used immediately or stored at $-80^{\circ}$. New FFPE fixed slides were not obtained for any cases, and subsequent reliability and reproducibility experiments were performed beginning with the cDNA generation and amplification step. Fresh samples were not processed to perform the algorithm software reliability studies.

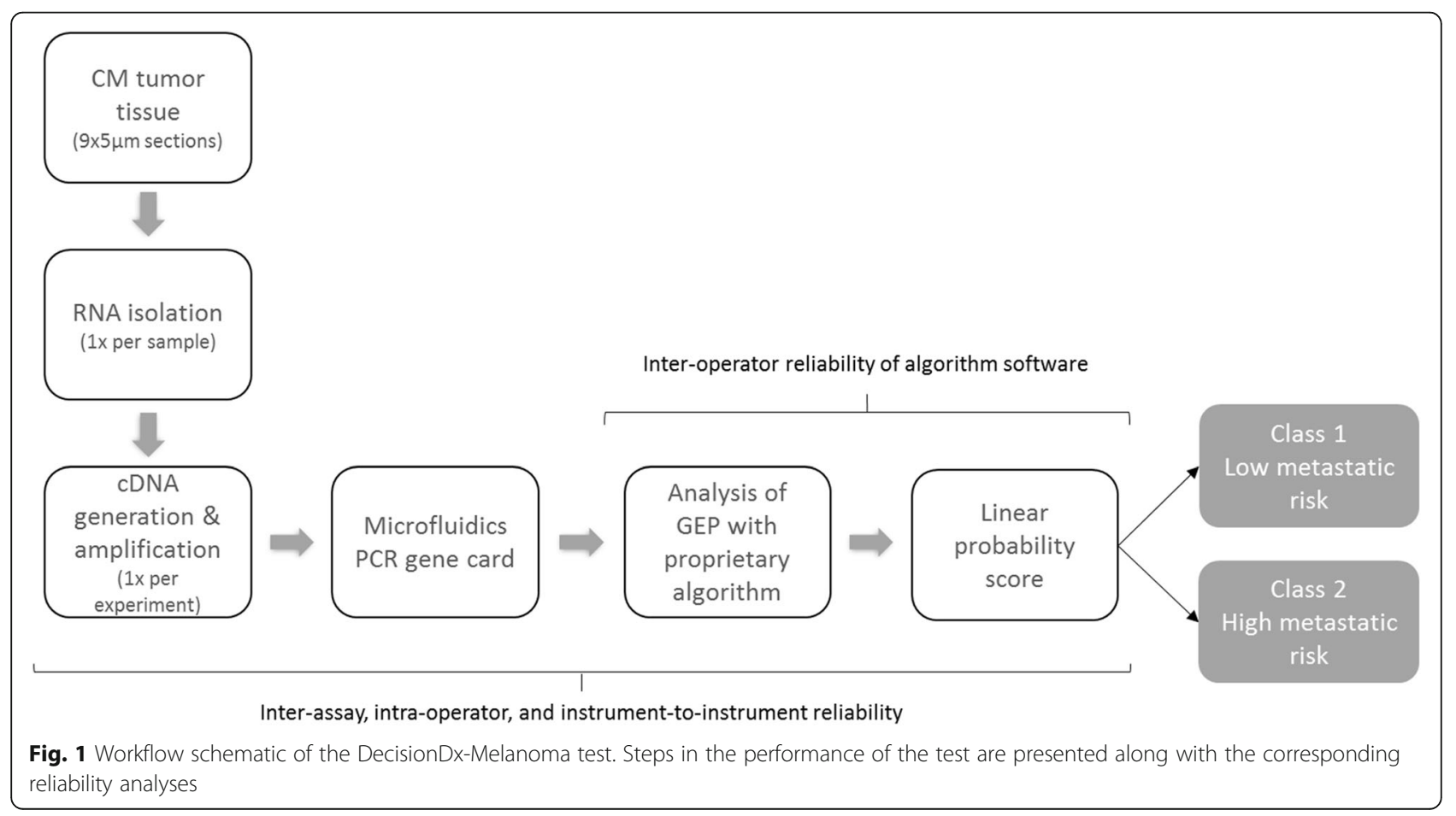


Resulting standardized $\Delta \mathrm{Ct}$ values for each test sample were analyzed with a radial basis machine (RBM) predictive model from a validated training set of 164 melanoma cases with known metastatic outcomes [9] using JMP Genomics SAS-based software (SAS, Cary, NC). RBM modeling provides a qualitative binary classification of Class 1 (low risk) or Class 2 (high risk) tumor biology based on a quantitative linear probability score from 0.0 to 1.0 , with a score of 0.5 being the cutoff between the binary classes. A normal confidence interval for each Class is established by using one SD from the median score of the training set cases without recurrence $(0.0-0.41)$ or with recurrence $(0.59-1.0)$; scores falling outside this range are considered of reduced statistical confidence $(0.41-0.5 ; 0.5-0.59)$. Results are reported as Class $1 \mathrm{~A}(0.0-0.41)$, Class $1 \mathrm{~B}(0.41-0.5)$, Class 2A (0.5-0.59) and Class 2B (0.59-1.0).

\section{Statistical analysis}

Statistical analysis was performed using Microsoft Excel and WinSTAT for Microsoft Excel version 2012.1 (R. Fitch Software, Cambridge, MA) and/or the R package. Analytic validity and reliability was reported as 1) the qualitative concordance of RBM binary class assignment (Class 1 or Class 2), 2) the qualitative concordance of $\mathrm{RBM}$ subclass assignment (Classes $1 \mathrm{~A}, 1 \mathrm{~B}, 2 \mathrm{~A}$ or $2 \mathrm{~B}$ ) and 3) correlation of quantitative probability score values. Association of clinical factors with test outcomes was primarily determined using Fisher's exact and $X$ - squared tests, with other tests indicated where appropriate.

\section{Results}

Specimen and sample stability

To assess RNA stability, results for 21 samples were obtained on 2 separate days, 3 months apart. Complimentary DNA was generated for each of the two experiments using the original isolated RNA sample. Comparison of probability score values (range $0.0-1.0$ ) showed high correlation $\left(\mathrm{R}^{2}=0.99, p<0.001\right), 100 \%$ concordance in binary risk classification (Class 1 or Class 2) and $90 \%$ concordance on subclass risk classification (Class 1A, 1B, 2A, or 2B) (18 of 21 cases; $95 \%$ confidence interval $[\mathrm{CI}]=70-99 \%$ ). Analysis was also performed on an additional 20 samples tested on 2 separate days at intervals ranging from 48 to 122 days apart. Again, probability score values were highly correlated $\left(\mathrm{R}^{2}=0.96, p<0.02\right)$ with $100 \%$ concordance $(95 \% \mathrm{CI}=$ $83-100 \%)$ in binary class assignment. Risk classification using normal and reduced confidence subclasses was concordant in 18 of 20 (90\%) cases (95\% CI = 68-99\%).

To evaluate long-term cDNA stability, we monitored reproducibility of assay performance for one Class 1 and one Class 2 cDNA sample (positive controls) included from experiment to experiment and across multiple lots of reagents (Fig. 2). Two negative water controls without template were also included with each OpenArray run over a 3-month period in which 56 assays were

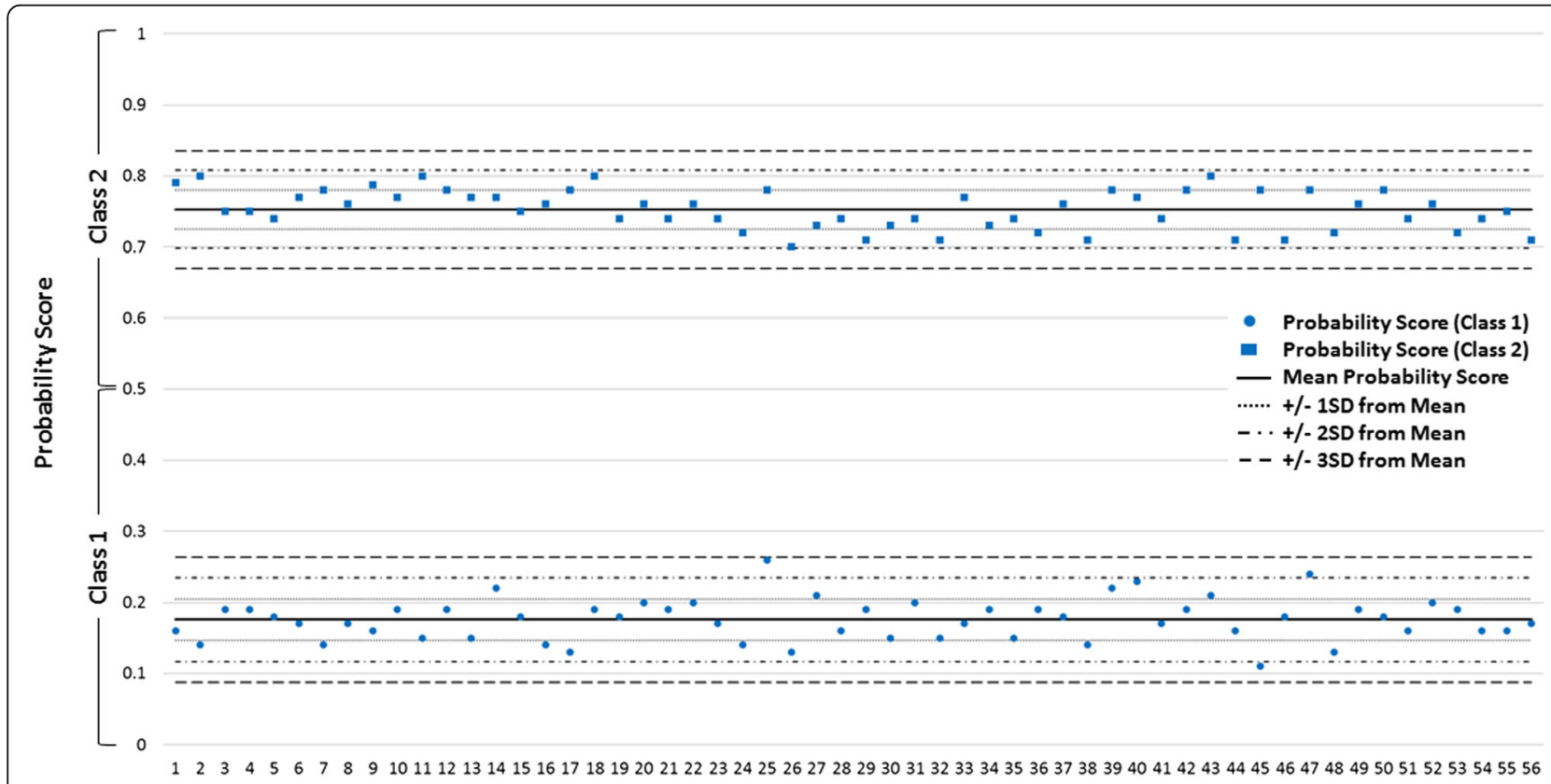

Fig. 2 Levey-Jennings analysis for positive control samples across 56 experiments. Reproducibility of assay performance for one Class 1 and one Class 2 positive control cDNA sample is shown. Probability scores were recorded from experiment to experiment over a 3 month period and across multiple lots of reagents 
performed. No assays were rejected due to amplification in the negative controls. The Class 1 positive control sample had a mean quantitative probability score of $0.176(\mathrm{SD}=0.029,2 \mathrm{SD}=0.059$ and $3 \mathrm{SD}=0.088)$ and the Class 2 positive control sample had a mean probability score of $0.752(\mathrm{SD}=0.027,2 \mathrm{SD}=0.055$, and $3 \mathrm{SD}=$ 0.082 ), reflecting robust assay repeatability.

Short-term cDNA stability was also evaluated. Ten samples underwent reverse transcription and the cDNA was stored for $96 \mathrm{~h}$ per standard operating procedures; RNA from the same 10 samples was then reverse transcribed on the day the assay was performed. All samples were run on a single assay and the resulting probability scores from the two groups were compared. We found probability score values to be significantly correlated $\left(\mathrm{R}^{2}\right.$ $=0.89, p<0.05)$, and both subclass and binary risk classifications were $100 \%$ concordant $(95 \% \mathrm{CI}=69-100 \%$ for both).

To further examine sample stability, the success rates of GEP processing at various time points after diagnosis were assessed. We examined a total of 6772 FFPEderived samples with documented age of specimen that were stored for up to 1 year, 1-2 years, 2-3 years, 34 years, or greater than 4 years prior to GEP testing. Overall we observed 98\% (6647 of 6772) success rate in all specimens. There was a slight decrease in success rates in samples that had been stored for longer periods of time ( $p<0.0001$; Fig. 3).

We also examined the effect of delay in sample processing on the stability of the 31-gene GEP assay. We evaluated outcomes in 275 retrospective research samples processed 1.5 to 16 years after diagnosis in which a significant association between GEP Class and recurrence-free survival has been previously published $[8,9]$. Multivariate Cox regression model to evaluate the interaction between GEP Class and time to sample processing showed no effect of delay in processing time ( $p$ $=0.25)$ and no statistical interaction between the sample age and GEP Class covariates $(p=0.51)$ was observed. These data indicate that the delay in processing time does not alter association of Class assignment and recurrence risk.

\section{DecisionDx-Melanoma assay reliability}

To assess inter-assay reliability of the 31-gene expression profile test, results were obtained on two separate days for 168 clinical melanoma samples. The time interval between the testing of matched samples ranged from 1 day to greater than 6 weeks. A total of 44 clinical samples were analyzed using the 7900HT Real-Time PCR System, and 124 samples were analyzed using the QuantStudio Real-Time PCR System. Comparison of probability score values (range $0.0-1.0$ ) resulted in highly correlated scores $\left(R^{2}=0.96, p<0.001\right.$; Fig. 4a). Binary risk classification was concordant for 167 of 168 (99\%, 95\% CI 96-100\%) cases and subclassification was concordant for 155 of 168 (92\%, 95\% CI 87-96\%) cases. The single case changing from Class 1 to 2 generated probability scores close to the 0.5 cutoff in the first run (0.476). Overall, the mean absolute difference in matched probability scores was 0.03 and showed $95 \%$ of variability to be within acceptable limits and not likely to change class assignment, as determined by Bland-Altman analysis (Fig. 4b).

We evaluated intra-assay reliability by obtaining results from 7 samples run in triplicate on a single OpenArray

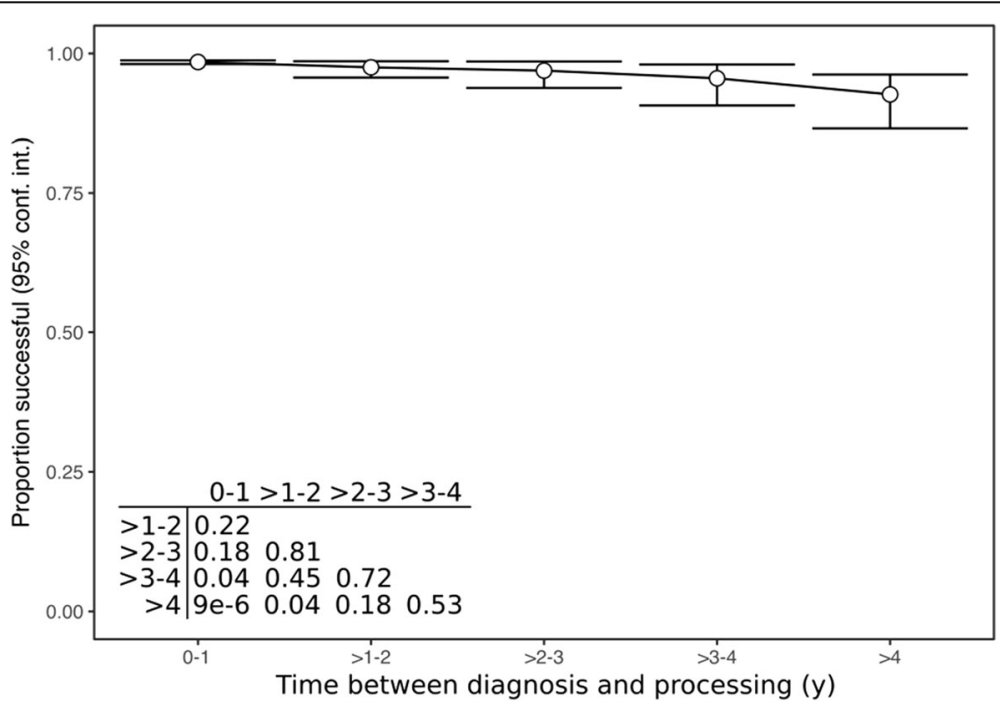

Fig. 3 Success rates for the DecisionDx-Melanoma test for FFPE samples at various time points after diagnosis. Processing times are in years since diagnosis. Error bars represent 95\% confidence intervals for the proportion of successful GEP tests in the indicated bin. Inset table represents the adjusted (FDR) $p$-values of Chi-squared comparisons between the indicated time bins 

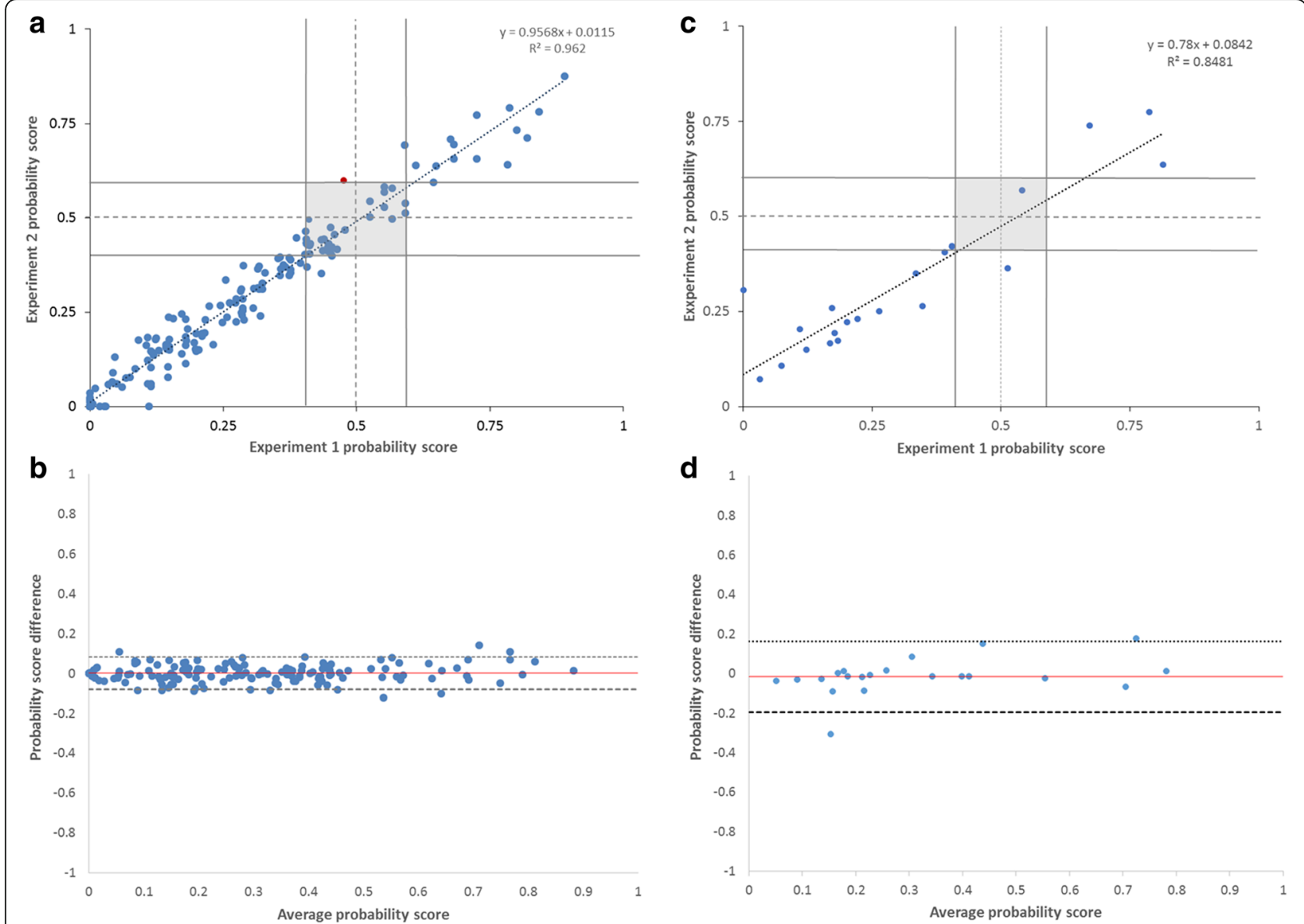

Fig. 4 Results from concordance studies on probability score values of the DecisionDx-Melanoma test. a Inter-assay correlation analysis for 168 cases; b Bland-Altman plot for 168 cases showing estimated bias (mean difference in probability scores, red line) and 95\% confidence interval (dashed lines); c instrument-to-instrument correlation analysis for 21 cases; $\mathbf{d}$ Bland-Altman plot for 21 cases showing estimated bias (mean difference in probability scores, red line) and 95\% confidence interval (dashed lines)

plate. The process was repeated on 3 separate runs for a total of 21 samples. Binary classification resulted in $100 \%$ concordance (95\% CI 94-100\%) while subclassification resulted in 98\% concordance (62 of 63; 95\% CI 91-100\%).

Lot to lot variability for critical reagents has been evaluated in experiments ranging from 4 to 19 samples and with 2-6 reagent lots. Correlation of discriminant scores was above 0.96 for all experiments, with binary class concordance of $100 \%$ in all cases and subclass concordance above $90 \%$ for all but one reagent, in which a subclass concordance of $75 \%$ was achieved based on only one of four samples being discrepant (Additional file 1: Table S1).

Inter-platform reliability was assessed by comparing probability scores generated from 21 samples tested on both the 7900HT and QuantStudio systems. The results indicated significant correlation of probability score values between the two systems $\left(R^{2}=0.85, p<0.001\right.$; Fig. $\left.4 c\right)$, and concordant subclass prediction was observed for $95 \%$ of cases (19 of 21). One of the matched probability score values generated for each of the two discordant cases was in the reduced confidence range $(0.421$, Class $1 \mathrm{~B}$ and 0.513 , Class 2A). The mean absolute difference in probability scores between instruments was 0.06 (Fig. 4d).

Twenty-two samples were run on two different QuantStudio instruments and the resulting probability scores were compared to evaluate inter-instrument reliability. Probability score values were highly correlated $\left(R^{2}=0.99\right.$, $p<0.001)$ and binary classification was concordant in 21 of 22 (95\%) cases (95\% CI 88-100\%). The mean absolute difference in probability score values between instruments was 0.02 .

\section{Inter-operator reproducibility of the predictive modeling algorithm}

To evaluate inter-operator reliability of the JMP Genomics predictive modeling software, RBM analysis of gene expression data for 268 clinically tested melanoma samples was performed separately by two personnel on multiple days. Quantitative probability scores generated by both analyses were identical $\left(\mathrm{R}^{2}=1.0, p<0.001\right.$; data not 
shown), and qualitative subclass and binary class prediction was concordant for all 268 cases (100\%).

\section{DecisionDx-Melanoma technical experience}

From March 1, 2013 through June 30, 2016, DecisionDx-Melanoma testing was requested for 8244 primary melanoma cases from 1123 centers in the United States and Spain. Samples submitted for DecisionDx-Melanoma testing must have a sufficient density of tumor cells in order to proceed with gene expression profiling. Of the 8244 specimens, 1221 (15\%) had insufficient tumor content for testing. As shown in Fig. 5, 90\% of the 1221 samples with insufficient tumor density were submitted during the period from March 1, 2013 to December 31, 2015, reflecting a 20\% rate of insufficient tissue for testing. Quality control studies completed in March 2015 permitted a decrease in the required tumor content from $\geq 60 \%$ to $\geq 40 \%$ melanoma within a macro-dissectible area of the tissue section. This, coupled with efforts to improve biopsy tissue preservation at the local processing level (including educational outreach to pathology laboratory staff, pathologists, and ordering clinicians), resulted in a dramatic reduction in the number of insufficient specimens. From January 1, 2016 to June 30, 2016, only 4.4\% (124 of 2806) of samples lacked sufficient tumor content, reflecting $78 \%$ reduction in quality control rejections compared to the previous period (Fig. 5). No changes in the proportion of thin tumor ( $\leq 1 \mathrm{~mm}$ Breslow thickness) cases was observed in this period.

Overall, 98\% (6895 of 7023) of cases submitted with sufficient tumor volume were successfully tested and reported, with only $1.8 \%$ cases having a reported technical failure due to amplification failure in control and/or prognostic genes. The technical success rate increased to
99\% (2647 of 2682) for the period of January 1, 2016 to June 30, 2016 (Fig. 5).

\section{Discussion}

As precision medicine in oncology strongly relies on accurate molecular classification of tumors, it becomes imperative to determine the reliability and accuracy of molecular tests. Groups such as the Evaluations of Genomic Applications in Practice and Prevention (EGAPP) Working Group and National Comprehensive Cancer Network (NCCN) have recognized three integral components of molecular diagnostic and prognostic tests: analytic validity, clinical validity, and clinical utility $[11,12]$. The GEP test described in this study has been clinically validated in three multicenter studies, showing that molecular class assignment is able to accurately and reliably identify CM patients who have a high risk of developing metastases $[8,9,13]$, and its clinical utility was recently reported in a study showing that physicians directed their management choices based on patients' GEP risk classification [14]. Here we aimed to report the analytic validity of the GEP using recognized measures of reliability.

Inter-assay, inter-operator, and inter-instrument reliability, measured using both the quantitative probability scores and binary classifications of risk, met or exceeded the requirements for a clinically applied prognostic test $[12,15,16]$. Probability scores were highly correlated when the same samples were tested on different days or experiments were performed on different machines $\left(R^{2}\right.$ $=0.96$ and 0.85 , respectively), and concordance of binary class prediction was strong for both analytic parameters (99\% and 95\%, respectively). These results highlight the strength of the protocols used to perform the 31-gene test, and the reproducibility of results when the test is

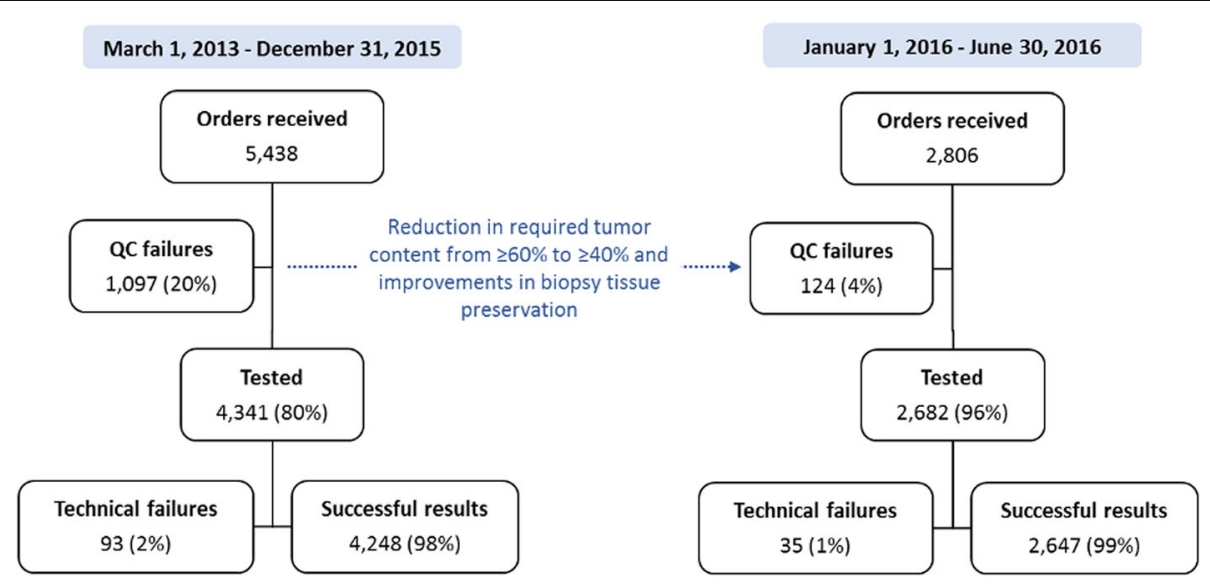

Fig. 5 Technical experience of the DecisionDx-Melanoma test for samples submitted from March 2013 to June 2016. Quality control rejections, technical failures and successfully tested samples are presented for the time periods before and after the reduction in required tumor density, implemented after successful clinical validation and through educational efforts to improve biopsy tissue preservation at the dermatopathology level 
run in a CAP-accredited, CLIA-certified central laboratory.

The majority of CM tumors are diagnosed when they are $<1 \mathrm{~mm}$ in thickness [6,7]. As such, the amount of tumor tissue for diagnostic and prognostic testing can be limited and preservation of that tissue is an important consideration in the management of $\mathrm{CM}$ patients. Tumor tissue preservation is increasingly a priority as biomarker testing, such as BRAF mutational analysis, PD-L1 immunohistochemistry and this GEP test, are integrated in patient management decisions [17-19]. The 98\% technical success rate of the test indicates consistent high performance using available tumor biopsy tissue and compares favorably to the performance of other genomic classifier tests performed on FFPE specimens [20]. This result is achieved despite the fact that clinical specimens were submitted by over 1100 institutions submitting clinical samples, and reflects robustness regardless of institution-specific tissue processing protocols and shipment variables. Our results also highlight the importance of communication between laboratories, as the number of specimens with insufficient tumor tissue was dramatically improved by implementing direct communication with dermatopathologists to improve biopsy tissue preservation measures. As shown in Fig. 5, the result is that $96 \%$ of submitted specimens were clinically tested from January 1 through June 30, 2016. As there is increased recognition of the value of molecular testing, it is important to develop sample preservation protocols and robust molecular tests that will enable the clinical application when limited tissue is available.

While there is expected correlation between the GEP prediction of risk and AJCC staging, there is no perfect correlation. We know that, despite having a low population-based risk, the majority of patients that die from melanoma are initially diagnosed with stage I or II disease [1, 5-7]. Previous studies have shown that the GEP test a) is independent from the standard clinicopathologic staging parameters, b) adds additional information about recurrence/metastasis risk and, c) is able to identify up to $90 \%$ of Stage I and II patients who die from their disease as high-risk (Class 2) [8, 9].

\section{Conclusions}

These results demonstrate that the 31-gene expression profile test is a precise, reliable and technically robust molecular test. Using a framework of accepted criteria to establish analytic validity, we present strong test performance and reproducibility. Taken together, the results of this analysis and previous clinical validation studies show that the GEP prognostic test is a robust and clinically useful tool to implement risk-appropriate healthcare decision-making in CM patients.

\section{Additional file}

Additional file 1: Table S1 Lot-to-lot stability of reagents used to run the DecisionDx-Melanoma test. (DOCX $14 \mathrm{~kb}$ )

\section{Abbreviations \\ AJCC: American Joint Committee on Cancer; CAP: College of American Pathologists; Cl: Confidence interval; CLIA: Clinical Laboratory Improvement Act; CM: Cutaneous melanoma; EGAPP: Evaluations of Genomic Applications in Practice and Prevention; FFPE: Formalin-fixed, paraffin-embedded; GEP: Gene expression profile; H\&E: Hematoxylin and eosin; NCCN: National Comprehensive Cancer Network; RBM: Radial basis machine; SD: Standard deviation; SLN: Sentinel lymph node; SLNB: Sentinel lymph node biopsy}

\section{Acknowledgements}

The authors wish to thank Trisha Poteet and Nathalie Lassen for their work in data collection and analysis.

\section{Funding}

This study was sponsored by Castle Biosciences, Inc.

Availability of data and materials

The dataset analysed during the current study is available from the corresponding author upon reasonable request.

\section{Authors' contributions}

Conceptualization: RWC, FAM; Validation: RWC; Formal analysis: RWC, BM, JW, KRC, FAM, JFS; Writing - original draft: RWC, BM; Writing - review \& editing: RWC, BM, JW, KRC, FAM, KO, JFS; Visualization: RWC, BM, JW, FAM, JFS; Supervision: RWC. All authors have read and approved this manuscript.

\section{Ethics approval and consent to participate}

Institutional review board approval was not required because this analysis is exempt from the regulatory review requirements as set forth in section 46.101 (b) of 45 CFR 46. Patient consent was not required given the retrospective nature of the study and the use of aggregate de-identified data.

Consent for publication

Not applicable.

\section{Competing interests}

All authors are employees of Castle Biosciences, Inc. and hold stock in the company.

\section{Publisher's Note}

Springer Nature remains neutral with regard to jurisdictional claims in published maps and institutional affiliations.

\section{Author details}

${ }^{1}$ Castle Biosciences, Inc., 820 S. Friendswood Dr., Suite 201, Friendswood, TX 77546, USA. ${ }^{2} 3737$ N. 7th St. \#160, Phoenix 85014, Phoenix, USA.

Received: 7 November 2017 Accepted: 2 February 2018 Published online: 13 February 2018

\section{References}

1. Balch CM, Gershenwald JE, Soong SJ, Thompson JF, Atkins MB, Byrd DR, et al. Final version of 2009 AJCC melanoma staging and classification. J Clin Oncol. 2009:27:6199-206.

2. Balch CM, Gershenwald JE, Soong SJ, Thompson JF, Ding S, Byrd DR, et al. Multivariate analysis of prognostic factors among 2,313 patients with stage III melanoma: comparison of nodal micrometastases versus macrometastases. J Clin Oncol. 2010:28:2452-9.

3. Gershenwald JE, Colome MI, Lee JE, Mansfield PF, Tseng C, Lee JJ, et al. Patterns of recurrence following a negative sentinel lymph node biopsy in 243 patients with stage I or II melanoma. J Clin Oncol. 1998;16:2253-60. 
4. Morton DL, Thompson JF, Cochran AJ, Mozzillo N, Nieweg OE, Roses DF, et al. Final trial report of sentinel-node biopsy versus nodal observation in melanoma. N Engl J Med. 2014;370:599-609.

5. Edge SB, Compton CC. AJCC Cancer Staging Manual 7th edition-melanoma. Ann Surg Oncol. 2010;17:1471147-4.

6. Whiteman DC, Baade PD, Olsen CM. More people die from thin melanomas $(1 \mathrm{~mm})$ than from thick melanomas (>4 mm) in Queensland, Australia. J Invest Dermatol. 2015;135:1190-3.

7. Shaikh WR, Dusza SW, Weinstock MA, Oliveria SA, Geller AC, Halpern AC. Melanoma Thickness and Survival Trends in the United States, 1989 to 2009 J Natl Cancer Inst. 2016;108:djv294.

8. Gerami P, Cook RW, Russell MC, Wilkinson J, Amaria RN, Gonzalez R, et al. Gene expression profiling for molecular staging of cutaneous melanoma in patients undergoing sentinel lymph node biopsy. J Am Acad Dermatol. 2015:72:780-5. e3

9. Gerami P, Cook RW, Wilkinson J, Russell MC, Dhillon N, Amaria RN, et al. Development of a prognostic genetic signature to predict the metastatic risk associated with cutaneous melanoma. Clin Cancer Res. 2015;21:175-83.

10. Sun F, Bruening W, Uhl S, Ballard R, Tipton K, Schoelles K. Quality, Regulation and Clinical Utility of Laboratory-developed Molecular Tests. Rockville, MD: Agency for Healthcare Research and Quality (US); 2010.

11. Engstrom PF, Bloom MG, Demetri GD, Febbo PG, Goeckeler W, Ladanyi M, et al. NCCN molecular testing white paper: effectiveness, efficiency, and reimbursement. J Natl Compr Cancer Netw. 2011;9(Suppl 6):S1-16.

12. Teutsch SM, Bradley LA, Palomaki GE, Haddow JE, Piper M, Calonge N, et al. The evaluation of genomic applications in practice and prevention (EGAPP) initiative: methods of the EGAPP working group. Genet Med. 2009;11:3-14.

13. Hsueh EC, DeBloom JR, Lee J, Sussman JJ, Covington KR, Middlebrook B, et al. Interim analysis of survival in a prospective, multi-center registry cohort of cutaneous melanoma tested with a prognostic 31-gene expression profile test. J Hematol Oncol. 2017;10:152

14. Berger AC, Davidson RS, Poitras JK, Chabra I, Hope R, Brackeen A, et al. Clinical impact of a 31-gene expression profile test for cutaneous melanoma in 156 prospectively and consecutively tested patients. Curr Med Res Opin. 2016:32:1599-604.

15. Cronin M, Sangli C, Liu ML, Pho M, Dutta D, Nguyen A, et al. Analytical validation of the Oncotype DX genomic diagnostic test for recurrence prognosis and therapeutic response prediction in node-negative, estrogen receptor-positive breast cancer. Clin Chem. 2007:53:1084-91.

16. Walsh PS, Wilde JI, Tom EY, Reynolds JD, Chen DC, Chudova DI, et al. Analytical performance verification of a molecular diagnostic for cytologyindeterminate thyroid nodules. J Clin Endocrinol Metab. 2012;97:E2297-306.

17. Lade-Keller J, Rømer KM, Guldberg P, Riber-Hansen R, Hansen LL, Steiniche $T$, et al. Evaluation of BRAF mutation testing methodologies in formalinfixed, paraffin-embedded cutaneous melanomas. J Mol Diagn. 2013;15:70-80.

18. Topalian SL, Hodi FS, Brahmer JR, Gettinger SN, Smith DC, McDermott DF, et al. Safety, activity, and immune correlates of anti-PD-1 antibody in cancer. N Engl J Med. 2012;366:2443-54.

19. Dietel M, Jöhrens K, Laffert MV, Hummel M, Bläker H, Pfitzner BM, et al. A 2015 update on predictive molecular pathology and its role in targeted cancer therapy: a review focussing on clinical relevance. Cancer Gene Ther. 2015;22:417-30.

20. Health Quality Ontario. Gene expression profiling for guiding adjuvant chemotherapy decisions in women with early breast cancer: an evidencebased and economic analysis. Ont Health Technol Assess Ser. 2010;10:1-57.

\section{Submit your next manuscript to BioMed Central and we will help you at every step:}

- We accept pre-submission inquiries

- Our selector tool helps you to find the most relevant journal

- We provide round the clock customer support

- Convenient online submission

- Thorough peer review

- Inclusion in PubMed and all major indexing services

- Maximum visibility for your research

Submit your manuscript at www.biomedcentral.com/submit 